\title{
Cardiac Profile of Filipino Patients With Liver Cirrhosis: A 10-Year Study
}

\author{
Emily Mae L. Yap ${ }^{\mathrm{a}, \mathrm{c}}$, Myla Gloria S. Supe ${ }^{\mathrm{a}}$, Ira I. Yu ${ }^{\mathrm{b}}$
}

\begin{abstract}
Background: Cardiac complications among patients with liver cirrhosis have not yet been described among Filipinos. Cirrhotic cardiomyopathy is a rarely described complication that has not been extensively described in literature. This is the first study to describe the electrocardiographic and echocardiographic findings of Filipino patients with liver cirrhosis.
\end{abstract}

Methods: A retrospective analytical study of 148 patients with liver cirrhosis from 2007 to 2016 at the Philippine Heart Center was done. The clinical characteristics, median QTc interval, systolic and diastolic functions on echocardiography of these patients were described. Spearman rho correlation was employed to determine the rank order correlation between QTc prolongation and the severity of liver cirrhosis. Fisher's Exact test was used to test the association of the echocardiographic parameters with the severity of liver cirrhosis.

Results: The 10-year prevalence rate of liver cirrhosis at the Philippine Heart Center was $0.001 \%(148 / 137,584)$. The mean age was $72.4 \pm$ 14 years with a female/male ratio of $1.1: 1$. The most common etiology of cirrhosis was hepatitis B or C infection $(20 \%, 29)$. The Child-Pugh Classification (CPC) and Model for End-Stage Liver Disease (MELD) score were used to determine the severity of liver cirrhosis and to assess their prognosis. There were 31 patients $(24 \%)$ with CPC-A, 84 patients (64\%) with CPC-B and 15 patients (11\%) with CPC-C. Fifty-five percent $(n=69)$ had a MELD score of 16 and below. Prolongation of the QTc interval was only seen among those with CPC A (median QTc of $470 \mathrm{~ms}$ ) and a MELD score of 9 and below (median QTc of $485 \mathrm{~ms}$ ). The mean left ventricular ejection fraction was $54.40 \pm 28.63 \%$. There were five patients with a left ventricular ejection fraction of $<55 \%$. The mean cardiac output $\left(6.04 \pm 5.24 \mathrm{~L} / \mathrm{min} / \mathrm{m}^{2}\right)$ and cardiac index $(2.92 \pm$ $1.47 \mathrm{~L} / \mathrm{min} / \mathrm{m}^{2}$ ) were normal. There were 44 patients who had evidence of diastolic dysfunction based on an $\mathrm{E} / \mathrm{A}$ ratio $<1$, prolongation of isovolumic relaxation time (IVRT) of $>80 \mathrm{~ms}$ and prolongation of deceleration time (DT) of $>200 \mathrm{~ms}$. There were only five patients who fulfilled

Manuscript submitted November 8, 2018, accepted November 19, 2018

aDepartment of Adult Cardiology, Philippine Heart Center, Quezon City, Philippines

${ }^{\mathrm{b}}$ Gastroenterology Unit, Department of Allied Medical Sciences, Philippine Heart Center, Quezon City, Philippines

${ }^{\mathrm{c} C}$ Corresponding Author: Emily Mae L. Yap, Department of Adult Cardiology, Philippine Heart Center, East Avenue, Quezon City, Philippines.

Email: emilyyap@gmail.com

doi: https://doi.org/10.14740/cr804 the criteria for cirrhotic cardiomyopathy. A majority of the patients were discharged improved (82\%). There were 26 cases who expired (18\%).

Conclusion: A higher mean age of Filipinos with liver cirrhosis was reported in our study. Prolongation of the QTc interval was seen among those with early and late stage of cirrhosis (CPC A or MELD score $\leq 9$ and $\mathrm{CPC}$ C). Most of these patients had normal left ventricular systolic function precluding the presence of cirrhotic cardiomyopathy.

Keywords: Liver cirrhosis; Cirrhotic cardiomyopathy; Chronic liver disease; Hepatitis

\section{Introduction}

Liver cirrhosis is the development of degenerative nodules surrounded by fibrous bands in response to chronic liver injury which may be brought about by chronic alcohol intake, hepatitis $\mathrm{B}$ or $\mathrm{C}$ infection, non-alcoholic steatohepatitis or other hepatic insults which may lead to end-stage liver disease [1]. Cirrhotic cardiomyopathy, a rarely described complication, is defined as the presence of chronic cardiac dysfunction among patients with liver cirrhosis which is characterized by an abnormal and blunted contractile responsiveness to physiological, pathological or pharmacological stress, in the absence of known cardiac disease and irrespective of the causes of cirrhosis [2, 3].

The actual prevalence of cirrhotic cardiomyopathy is unknown [2]. Most of these patients have normal or near normal cardiac function at rest but with abnormal cardiac responses during exertion, stress or liver transplantation [1-3]. The natural history of the disease has not been extensively studied yet. The condition has been described to be well tolerated with most patients being asymptomatic during the initial development of this complication [2].

The majority of patients are diagnosed during phases of clinical decompensation of cirrhosis in which they present with features of diastolic heart failure and/or high-output heart failure [4]. Impaired cardiac function is often undiagnosed in liver cirrhosis, but this increases the mortality risk in the setting of acute decompensated cirrhosis [5].

Cardiac complications seen among patients with liver cirrhosis have not been extensively studied. Clinical trials on this subset of patients with liver cirrhosis who have developed cardiomyopathy are still lacking. There continues to be no accepted pharmacologic treatment for cirrhotic cardiomyopathy 
other than the guidelines set forth by the American College of Cardiology/American Heart Association guidelines for the treatment of heart failure $[6,7]$.

To date, there have been no studies of this complication of liver cirrhosis in our country, making its incidence and prevalence unknown. This is the first published study describing the electrocardiographic and echocardiographic findings of Filipino adults with liver cirrhosis. This is aimed to increase awareness of cirrhotic cardiomyopathy and to prompt physicians to include cardiac work-up among their patients with liver cirrhosis.

\section{Patients and Methods}

\section{Study design}

This was a retrospective analytical study conducted at the Philippine Heart Center (PHC), a tertiary referral center, located at East Avenue, Quezon City, from January 1, 2017 to December 31,2017 . This was conducted in compliance with the ethical principles set forth in the Declaration of Helsinki. Prior to the study initiation, the protocol was reviewed and approved by the PHC Institutional Ethics Review Board.

\section{Study population}

All patients $\geq 19$ years old who were diagnosed with liver cirrhosis from January 1, 2007 to December 31, 2016 were included in this study. Patients with concomitant cardiac problems such as but not limited to congenital heart disease, valvular heart disease, ischemic heart disease, hypertrophic cardiomyopathy and channelopathies were excluded.

\section{Study maneuver}

A retrospective analytical review of the records of 210 patients admitted at PHC with a final diagnosis of liver cirrhosis from 2007 to 2016 was done. The medical records were reviewed using the electronic document viewer and the actual charts of the patients at the medical records section. There were 62 patients excluded because they did not meet the inclusion criteria. The final analysis included 148 patients. Confidentiality of patient information was upheld as in the Declaration of Helsinki at all times. All data were encoded in password-protected Microsoft Excel spreadsheets. A code number was assigned to each patient. To maintain anonymity, a separate spreadsheet that linked the study code to the patient's name was made. Only the primary investigator had access to the file.

\section{Definition of terms}

\section{Liver cirrhosis}

It is an advanced stage of liver fibrosis (defined as the encap- sulation or replacement of injured tissue by a collagenous scar) that is accompanied by distortion of the hepatic vasculature. It leads to shunting of the portal and arterial blood supply directly [1].

\section{Cirrhotic cardiomyopathy}

At the 2005 World Congress of Gastroenterology at Montreal, Canada, the following diagnostic and supportive criteria for cirrhotic cardiomyopathy were proposed: 1) systolic dysfunction: blunted increase in cardiac output on exercise, volume challenge or pharmacological stimuli or resting ejection fraction $<55 \%$; 2) diastolic dysfunction: the ratio of early to late (atrial) phases of ventricular filling or E/A ratio $<1.0$ (age-corrected), prolonged deceleration time $(>200 \mathrm{~ms})$ or prolonged isovolumetric relaxation time (>80 ms);3) supportive criteria: electrophysiological abnormalities, abnormal chronotropic response, electromechanical uncoupling/dyssynchrony, prolonged QTc interval, enlarged left atrium, increased myocardial mass, increased brain natriuretic peptide (BNP), pro-BNP or troponin I [9].

\section{Child-Pugh Classification (CPC)}

This classification is used to assess the prognosis of chronic liver disease, mainly cirrhosis, as seen in Table 1. Although it was originally used to predict mortality during surgery, it is now used to determine the prognosis, the required strength of treatment and the necessity of liver transplantation [10].

Patients who are classified as CPC-A (5 - 6 points), CPC-B (7 - 9 points) and CPC-C (10 - 15 points) had a 1 -year survival of $100 \%, 81 \%$ and $45 \%$ respectively and a 2 -year survival of $85 \%, 57 \%$ and $35 \%$ respectively [10].

\section{Model for End-Stage Liver Disease (MELD) score}

It is a scoring system used to estimate prognosis and assess the severity of chronic liver disease and in determining prognosis and prioritizing receipt of a liver transplant. This is used by the United Network for Organ Sharing (UNOS) for prioritizing allocation of liver transplants instead of the older CPC score. This system uses the patient's values for serum bilirubin, serum creatinine and the international normalized ratio for prothrombin time (INR) to predict survival. It is calculated according to the following formula: MELD $=3.78 \times \ln$ (serum bilirubin $(\mathrm{mg} /$ $\mathrm{dL}))+11.2 \times \ln (\mathrm{INR})+9.57 \times \ln ($ serum creatinine $(\mathrm{mg} / \mathrm{dL}))+$ 6.43 . Scores are reported as whole numbers, so the result of the equation is rounded. Only the original, pre-2016, MELD score was used which excludes the serum sodium level [11].

\section{Definition of outcomes}

The primary outcome of our study were the electrocardiographic and echocardiographic features of patients with liver 
Table 1. Child-Pugh Classification of the Severity of Cirrhosis

\begin{tabular}{llll} 
& \multicolumn{2}{c}{ Child-Pugh Classification (CPC) } \\
\cline { 2 - 4 } & $\mathbf{1}$ & $\mathbf{2}$ & $\mathbf{3}$ \\
\hline Encephalopathy & None & Grades 1 - 2 (or precipitant-induced) & Grades 3-4 (or chronic) \\
Ascites & None & Mild/moderate (diuretic-responsive) & Severe (diuretic-refractory) \\
Bilirubin (mg/dL) & $<2$ & $2-3$ & $>3$ \\
Albumin (g/dL) & $>3.5$ & $2.8-3.5$ & $<2.8$ \\
PT $(\mathrm{s})$ & $<4$ & $4-6$ & $>6$ \\
INR & $<1.7$ & $1.7-2.3$ & $>2.3$ \\
\hline
\end{tabular}

cirrhosis. Secondary outcomes were the prevalence rate and the severity of liver cirrhosis.

\section{Statistical analysis}

Categorical variables such as demographics, comorbid illnesses, etiology of cirrhosis, outcome, electrocardiographic and echocardiographic features were described using frequency and percentages. Continuous variables were described using mean and standard deviation. The Spearman rho correlation was employed to determine the rank order correlation between QTc prolongation and the severity of liver cirrhosis. Fisher's Exact test was used to test the association of the electrocardiographic and echocardiographic parameters with the severity of liver cirrhosis.

\section{Results}

There were 148 admitted patients with liver cirrhosis in our institution from 2007 to 2016. The 10-year prevalence rate of liver cirrhosis was $0.001 \%(148 / 137,584)$. The mean age was $72.4 \pm 14$ years with a female/male ratio of $1.1: 1$. The mean duration of hospitalization was $7.5 \pm 6$ days. The most common etiology of cirrhosis was hepatitis B or C infection $(20 \%$, 29). The other etiologies can be seen in Table 2. A majority of the patients were discharged improved (82\%). There were 26 cases who expired (18\%).

The CPC and MELD score were used to determine the severity of chronic liver disease as seen in Table 3. Most of the patients in our cohort belonged to CPC B $(64 \%, 84)$. Most of the patients were discharged improved $(82 \%, 122)$.

There was no significant difference in the median QTc intervals based on their CPC, MELD score and outcome as shown in Table 4. Prolongation of the QTc interval was only seen among those with CPC A (median QTc: $470 \mathrm{~ms}$ ), CPC C (median QTc: $447 \mathrm{~ms}$ ) and a low MELD score of 9 and below (median QTc: $485 \mathrm{~ms}$ ).

Echocardiography was done in only 49 patients. Among these patients, there were only five patients who fulfilled the criteria for cirrhotic cardiomyopathy. There were five patients with a left ventricular ejection fraction of $<55 \%$. Table 5 shows the chamber dimensions and other echocardiographic findings. Notably, the mean left ventricular ejection fraction was $54.40 \pm 28.63 \%$. The mean cardiac output $(6.04 \pm 5.24 \mathrm{~L} /$ $\left.\mathrm{min} / \mathrm{m}^{2}\right)$ and cardiac index $\left(2.92 \pm 1.47 \mathrm{~L} / \mathrm{min} / \mathrm{m}^{2}\right)$ were normal. Among the echocardiographic parameters, there was a significant difference in the left ventricular end-systolic diameter based on body surface area (LVESD/BSA) $(\mathrm{P}=0.023)$ and cardiac output $(\mathrm{CO})(\mathrm{P}=0.003)$ between the different classes of $\mathrm{CPC}$. The diastolic function among patients with liver cirrhosis was also determined and there was a significant difference in the E/A ratio and systolic pulmonary artery pressure (SPAP) among the different classes of CPC as seen in Table 5. There were 44 patients who had evidence of diastolic dysfunction based on E/A ratio, isovolumic relaxation time (IVRT) and deceleration time (DT). Thirty-one patients had both prolonged DT and prolonged IVRT. There were seven patients who had

Table 2. General Characteristics of Patients Admitted With Liver Cirrhosis (2007 - 2016)

\begin{tabular}{|ll}
\hline Demographics & $\mathbf{f}(\%)$, mean \pm SD \\
\hline No. of patients & 148 \\
Age (years) & $72.4 \pm 14$ \\
Male & $70(47 \%)$ \\
Female & $78(53 \%)$ \\
Mean duration of hospitalization (days) & $7.5 \pm 6$ \\
Etiology of cirrhosis & \\
\hline Hepatitis & $30(20 \%)$ \\
$\quad$ Hepatitis B infection & $24(16 \%)$ \\
$\quad$ Hepatitis C infection & $6(4 \%)$ \\
Cryptogenic & $29(20 \%)$ \\
Non-alcoholic steatohepatitis & $27(18 \%)$ \\
Alcohol-related & $23(15 \%)$ \\
Schistosomiasis & $4(3 \%)$ \\
Hemosiderosis/hemochromatosis & $2(1 \%)$ \\
Autoimmune hepatitis & $1(0.7 \%)$ \\
Portal vein thrombosis & $1(0.7 \%)$ \\
Wilson's disease & $1(0.7 \%)$ \\
Outcome & $122(82 \%)$ \\
Discharged improved & $26(18 \%)$ \\
\hline Expired &
\end{tabular}


Table 3. Child Pugh Score (CPC), $n=130$

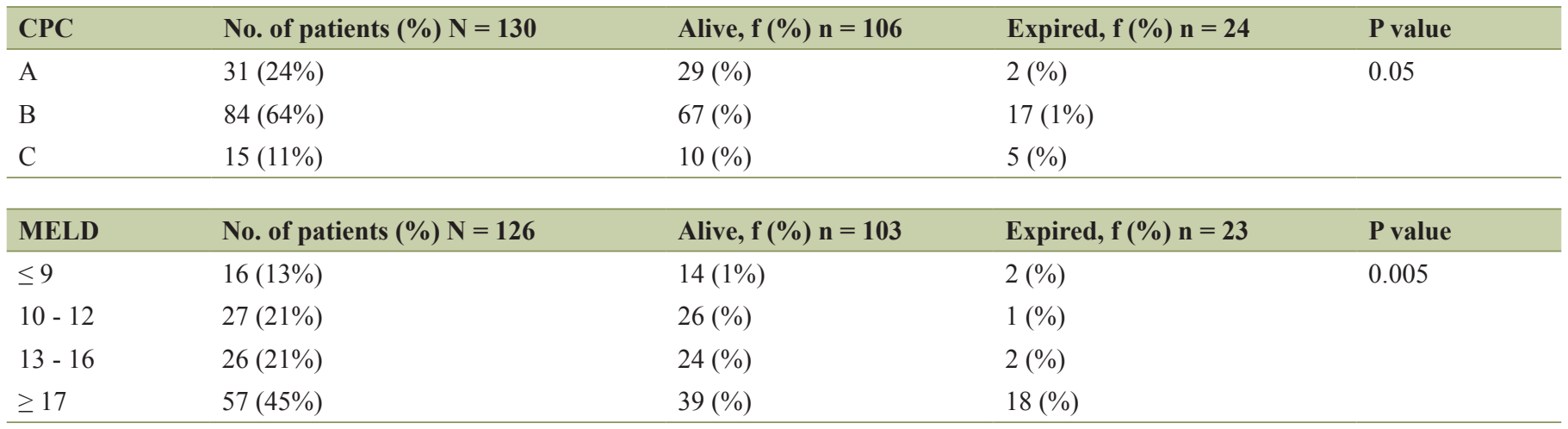

only prolonged IVRT. There were only six patients who had an abnormal E/A ratio of $<1$. Using the latest guidelines of the American Society of Echocardiography in the evaluation of left ventricular diastolic function, most of the patients had mild diastolic dysfunction (impaired relaxation) $(17,39 \%)$.

\section{Discussion}

This is the first study describing the cardiac profile of Filipinos with liver cirrhosis. The mean age of our patients was $72.4 \pm 14$ years with a slight female preponderance. This is significantly higher compared to studies done in other countries which reported lower mean ages $[12,13]$. Kumar et al [12] studied 90 patients with liver cirrhosis and reported a lower mean age of $46.2 \pm 10.8$ years and a male predominance $(78 \%)$. Bhatti et al [13] reported a mean age of $57.05 \pm 12.03$ years and a male predominance $(58 \%)$ in his cohort of 166 patients. The authors believed that this was related to the age of onset of the inciting factor that lead to the development of cirrhosis. Hepatitis B or
C infection was the most common cause in our study. This was an expected finding since hepatitis B infection is known to be hyperendemic in our country. A study by Wong et al reported an HBsAg seroprevalence rate of 16.7\% among Filipinos [14]. This corresponded to about 7.3 million Filipinos with chronic hepatitis B infection [14]. In another study, it was estimated that about $1 \%$ of the Filipinos have hepatitis $\mathrm{C}$ infection [15]. Gender differences in the prevalence of hepatitis B infection have not been reported [14]. This possibly explains the lack of significant gender predominance in our study. The seroprevalence of HBsAg is expected to peak between 20 to 39 years old [14]. Since cirrhosis takes years or decades to develop, this may explain why the patients in our study were older. Alcohol abuse $(61 \%, 55)$ was the most common cause of cirrhosis in a study done in Chennai [12]. This was the cause of cirrhosis in only $15 \%$ of our patients.

Compared to our cohort which only had 15 patients (11\%) classified as $\mathrm{CPC} \mathrm{C}$, there were more cases of CPC $\mathrm{C}$ in the study of Kumar et al $(49 \%, 36)$ and Bhatti et al $(45 \%, 77)$ which are reflective of a more decompensated state $[12,13]$.

Table 4. Median QTc Among the Different Child-Pugh Classification (CPC) and MELD Classification $(\mathrm{N}=84)$

\begin{tabular}{|c|c|c|c|c|c|}
\hline \multirow{2}{*}{$\mathrm{CPC}$} & \multirow{2}{*}{ No. of patients $(\%)$} & \multicolumn{3}{|c|}{ Median QTc, ms (IQR) } & \multirow{2}{*}{ P value } \\
\hline & & Total & Alive & Expired & \\
\hline A & $17(20 \%)$ & $470(423-501)$ & $470(412-496)$ & 607 & 0.152 \\
\hline $\mathrm{C}$ & $9(11 \%)$ & $447(412-484)$ & $\begin{array}{l}432(402-516) \\
0.117\end{array}$ & $\begin{array}{l}465(447-484) \\
0.256\end{array}$ & 0.462 \\
\hline \multirow{2}{*}{ MELD } & \multirow{2}{*}{ No. of patients (\%) } & \multicolumn{3}{|c|}{ Median QTc, ms (IQR) } & \multirow{2}{*}{ P value } \\
\hline & & Total & Alive & Expired & \\
\hline$\leq 9$ & $9(11 \%)$ & $485(436-501)$ & $488(370-607)$ & $485(436-501)$ & 0.769 \\
\hline $10-12$ & $16(20 \%)$ & $437(411-470)$ & 0 & $437(411-470)$ & \\
\hline Total & 82 & 439 & & & \\
\hline
\end{tabular}


Table 5. Echocardiographic Features in Patients With Liver Cirrhosis

\begin{tabular}{|c|c|c|c|c|c|c|}
\hline \multirow{2}{*}{ Parameter } & \multirow{2}{*}{ Normal range } & \multirow{2}{*}{ Mean \pm SD $(\mathrm{cm})$} & \multicolumn{3}{|c|}{ Child-Pugh Score } & \multirow{2}{*}{ P value } \\
\hline & & & $\mathbf{A}$ & B & $\mathbf{C}$ & \\
\hline LVEDD & & $4.28 \pm 0.91$ & $4.22 \pm 1.11$ & $4.36 \pm 0.81$ & $3.73 \pm 1.15$ & 0.445 \\
\hline LVESD & & $2.5 \pm 0.73$ & $2.70 \pm 1.12$ & $2.53 \pm 0.60$ & $2.07 \pm 0.68$ & 0.055 \\
\hline LVESD/BSA & $1.4-2.1 \mathrm{~cm} / \mathrm{m}^{2}$ & $1.51 \pm 0.44$ & $1.65 \pm 0.64$ & $1.46 \pm 0.38$ & $1.5 \pm 0.10$ & 0.023 \\
\hline IVSS & & $1.44 \pm 0.30$ & $1.40 \pm 0.32$ & $1.45 \pm 0.32$ & $1.33 \pm 0.11$ & 0.353 \\
\hline PWD & $\begin{array}{l}\text { F: } 0.6-0.9 \mathrm{~cm} \\
\text { M: } 0.6-1.0 \mathrm{~cm}\end{array}$ & $1.17 \pm 0.29$ & $1.23 \pm 0.36$ & $1.18 \pm 0.27$ & $1.13 \pm 0.32$ & 0.608 \\
\hline PWS & & $26.89 \pm 46.74$ & $34.26 \pm 57.29$ & $24.62 \pm 44.29$ & $41.43 \pm 68.91$ & 0.473 \\
\hline LA & $1.5-2.3 \mathrm{~cm} / \mathrm{m}^{2}$ & $3.61 \pm 1.00$ & $3.60 \pm 0.85$ & $3.68 \pm 0.944$ & $3.50 \pm 1.28$ & 0.748 \\
\hline LA/BSA & & $10.47 \pm 15.69$ & $13.4 \pm 20.01$ & $9.69 \pm 14.89$ & $8.73 \pm 10.5$ & 0.426 \\
\hline LAVI & $<28 \mathrm{~mL} / \mathrm{m}^{2}$ & $41.54 \pm 16.5$ & $44.17 \pm 18.20$ & $41.30 \pm 17.11$ & $47.33 \pm 19.10$ & 0.960 \\
\hline LVEF & $>55 \%$ & $54.40 \pm 28.63$ & $44.56 \pm 27.95$ & $57.02 \pm 28.34$ & $50.3 \pm 36.2$ & 0.883 \\
\hline $\mathrm{CO}$ & $>4.5 \mathrm{~L} / \mathrm{min}$ & $6.04 \pm 5.24$ & $5.06 \pm 2.31$ & $6.61 \pm 6.34$ & $4.40 \pm 1.47$ & 0.003 \\
\hline $\mathrm{CI}$ & $2.5 \mathrm{~L} / \mathrm{min} / \mathrm{m}^{2}$ & $2.92 \pm 1.47$ & $2.52 \pm 1.40$ & $3.08 \pm 1.41$ & $2.17 \pm 1.33$ & 0.993 \\
\hline
\end{tabular}

LVEDD: left ventricular end-diastolic dimension; BSA: body surface area; LVESD: left ventricular end-systolic diameter; IVSD: interventricular septum at end diastole; IVSS: interventricular septal end-systolic thickness; PWD: posterior wall thickness at end-diastole; PWS: posterior wall thickness at end-systole; LVMI: left ventricular mass index; RWT: relative wall thickness; LA: left atrium; LAVI: left atrial volume index; LVEF: left ventricular ejection fraction; CO: cardiac output; $\mathrm{Cl}$ : cardiac index.

Most of our patients were classified as CPC A $(20 \%, 17)$ and CPC B $(68 \%, 57)$ which have better prognosis. The authors postulated that the more severe degree of cirrhosis reported in the aforementioned studies could be due to the earlier age of onset.

The prevalence of cirrhotic cardiomyopathy could not be determined in this retrospective study due to the limited sample size and the incomplete diagnostics that were done. Among those with transthoracic echocardiography results, the test was only done at rest which has limited the full evaluation of systolic function. Stress echocardiography remains to be the recommended test to determine the presence of systolic dysfunction which is exemplified by a blunted increase in cardiac output with exercise, volume challenge or pharmacological stimuli [9].

Diastolic dysfunction was determined based on the E/A ratio, DT and IVRT. These were the same parameters used to evaluate diastolic function in the criteria for diagnosing cirrhotic cardiomyopathy in the expert consensus committee at the World Congress of Gastroenterology in Montreal, Canada in 2005 which has not been superceded as of this writing [9]. Prolonged DT was the most common abnormal parameter indicating diastolic dysfunction in our study. This is comparable to the findings reported by Alexander et al wherein prolonged
DT was a significant finding among those with alcoholic and non-alcoholic liver cirrhosis $(235.03 \pm 44.23 \mathrm{~ms}$ and $255.87 \pm$ $46.16 \mathrm{~ms}$, respectively) compared to healthy controls (185.83 $\pm 25.04 \mathrm{~ms}$ ) [16]. This indicates the presence of diastolic dysfunction in cirrhotic patients.

However, it is important to note that these criteria have not been updated for more than a decade [9] and other parameters that can better evaluate diastolic function are now being recommended. Specifically, the American Society of Echocardiography has updated their guidelines in the evaluation of left ventricular diastolic function and other parameters have been utilized to determine if left ventricular function is normal or abnormal [17]. The four recommended variables and their abnormal cutoff values are annular e' velocity (septal e' $<7 \mathrm{~cm} / \mathrm{s}$, lateral e' $<10 \mathrm{~cm} / \mathrm{s}$ ), average E/e' ratio $>14$, left atrial volume index $>34 \mathrm{~mL} / \mathrm{m}^{2}$ and peak tricuspid regurgitant velocity $>$ $2.8 \mathrm{~m} / \mathrm{s}$ [17]. Left ventricular diastolic function is normal if more than half of the available variables do not meet the cutoff values for identifying abnormal function. Left ventricular diastolic dysfunction is present if more than half of the available parameters meet these cutoff values [17]. The study is said to be inconclusive if half of the parameters do not meet the cutoff values. Using these new guidelines, 17 of our patients had grade 1 (impaired relaxation) diastolic dysfunction, while 10 
had grade 2 (pseudonormal pattern) or grade 3 (restrictive pattern) diastolic dysfunction.

Other supporting evidence for the presence of cirrhotic cardiomyopathy include electrophysiological abnormalities, abnormal chronotropic response, electromechanical uncoupling/dyssynchrony, prolonged QTc interval, enlarged left atrium, increased myocardial mass, increased BNP, pro-BNP or troponin I. Only the QTc interval, presence of left atrial enlargement as measured by the left atrial volume index (LAVI) and myocardial mass as measured in the left ventricular wall thickness were evaluated in our study.

Prolonged QTc interval has been reported to be an electrophysiological hallmark of cirrhotic cardiomyopathy [13]. Although studies have reported a correlation of the prolongation of the QT interval to the severity of liver cirrhosis, our patients were observed to have prolonged QT interval in the early stages of liver cirrhosis (CPC A or MELD score of 9 or below) and in the late stage of cirrhosis (CPC C). Most of the patients in our cohort belonged to CPC B. The median QTc interval of these patients was normal.

To overcome the inherent limitations of our study, we recommend a prospective multicenter study with the use of stress echocardiography and inclusion of all the recommended parameters to evaluate diastolic function to determine the full extent of cardiac dysfunction among those with liver cirrhosis.

\section{Conclusions}

A higher mean age of Filipinos with liver cirrhosis was reported in our study. Prolongation of the QTc interval was seen only among those with compensated liver cirrhosis (CPC A or MELD score of 9 and below). Most of these patients had normal left ventricular systolic function precluding the presence of cirrhotic cardiomyopathy.

\section{Conflict of Interest}

None.

\section{References}

1. Schuppan D, Afdhal NH. Liver cirrhosis. Lancet. 2008;371(9615):838-851.

2. Zardi EM, Abbate A, Zardi DM, Dobrina A, Margiotta D, Van Tassell BW, Afeltra A, et al. Cirrhotic cardiomyopathy. J Am Coll Cardiol. 2010;56(7):539-549.

3. Ripoll C, Catalina MV, Yotti R, Olmedilla L, Perez-Pena J, Lo Iacono O, Rincon D, et al. Cardiac dysfunction during liver transplantation: incidence and preoperative predictors. Transplantation. 2008;85(12):1766-1772.

4. Pozzi M, Carugo S, Boari G, Pecci V, de Ceglia S, Maggiolini S, Bolla GB, et al. Evidence of functional and structural cardiac abnormalities in cirrhotic patients with and without ascites. Hepatology. 1997;26(5):1131-1137.

5. Zambruni A, Trevisani F, Caraceni P, Bernardi M. Cardiac electrophysiological abnormalities in patients with cirrhosis. J Hepatol. 2006;44(5):994-1002.

6. Pudil R, Pelouch R, Praus R, Vasatova M, Hulek P. Heart failure in patients with liver cirrhosis. Cor et Vasa. 2013;55:e391-e396.

7. Jessup M, Abraham WT, Casey DE, et al. 2009 focused update: ACCF/AHA guidelines for the diagnosis and management of heart failure in adults: a report of the American College of Cardiology Foundation/American Heart Association Task Force on Practice Guidelines developed in collaboration with the International Society for Heart and Lung Transplantation. J Am Coll Cardiol. 2009;53:1343-1382.

8. Cesari M, Frigo AC, Tonon M, Angeli P. Cardiovascular predictors of death in patients with cirrhosis. Hepatology. 2018;68(1):215-223.

9. Gassanov N, Caglayan E, Semmo N, Massenkeil G, Er F. Cirrhotic cardiomyopathy: a cardiologist's perspective. World J Gastroenterol. 2014;20(42):15492-15498.

10. Cholongitas E, Papatheodoridis GV, Vangeli M, Terreni N, Patch D, Burroughs AK. Systematic review: The model for end-stage liver disease - should it replace ChildPugh's classification for assessing prognosis in cirrhosis? Aliment Pharmacol Ther. 2005;22(11-12):1079-1089.

11. Kamath PS, Kim WR, Advanced Liver Disease Study G. The model for end-stage liver disease (MELD). Hepatology. 2007;45(3):797-805

12. Kumar SS, Rajasigamani A, Socrates. Prevalence of cirrhotic cardiomyopathy in patients with cirrhosis of the liver: a tertiary hospital experience. Int J Pharm Bio Sci. 2017;8(1):(B)298-303.

13. Bhatti AB, Ali F, Satti SA. Prolonged QTc interval is an electrophysiological hallmark of cirrhotic cardiomyopathy. Open J Intern Med. 2014;4:33-39.

14. Wong SN, Ong JP, Labio ME, Cabahug OT, Daez ML, Valdellon E, Sollano JD, et al. Hepatitis B infection among adults in the philippines: A national seroprevalence study. World J Hepatol. 2013;5(4):214-219.

15. Yanase Y, Ohida T, Kaneita Y, Agdamag DM, Leano PS, Gill CJ. The prevalence of HIV, HBV and HCV among Filipino blood donors and overseas work visa applicants. Bull World Health Organ. 2007;85(2):131-137.

16. Alexander J, Mishra P, Desai N, Ambadekar S, Gala B, Sawant P. Cirrhotic cardiomyopathy: Indian scenario. J Gastroenterol Hepatol. 2007;22(3):395-399.

17. Nagueh SF, Smiseth OA, Appleton CP, Byrd BF, 3rd, Dokainish H, Edvardsen T, Flachskampf FA, et al. Recommendations for the evaluation of left ventricular diastolic function by echocardiography: an update from the American Society of Echocardiography and the European Association of Cardiovascular Imaging. J Am Soc Echocardiogr. 2016;29(4):277-314. 